\title{
Ansamitocin-induced Synchrony in Tetrahymena pyriformis
}

\author{
By SEIICHI TANIDA, * EIJI HIGASHIDE AND \\ MASAHIKO YONEDA \\ Microbiological Research Laboratories, Central Research Division, Takeda Chemical \\ Industries Ltd, 17-85, Jusohonmachi 2-chome, Yodogawa-ku, Osaka 532, Japan
}

(Received 4 October 1979; revised 19 December 1979)

\begin{abstract}
Ansamitocins inhibited cell division in Tetrahymena pyriformis $\mathrm{W}$ at low concentrations and caused cells to become more rounded. Exposure to ansamitocins for $5 \mathrm{~h}$ or more resulted in a burst of synchronous division at 100 min after removal of the antibiotics with a maximum division index of $70 \%$. A second burst of synchronous division occurred $300 \mathrm{~min}$ after removal with a peak division index of $55 \%$. The rounding of the cell shape was restored by the completion of the first division. When RNA or protein synthesis was blocked by chromomycin $A_{3}$ or cycloheximide, the first division was suppressed. When DNA synthesis was blocked by methotrexate plus uridine, synchronous division still occurred; furthermore, the DNA content of $T$. pyriformis cells did not increase before the first division. These findings suggest that the first synchronous division requires RNA and protein synthesis but does not require DNA synthesis. Interference by ansamitocins with the function of microtubule systems in $T$. pyriformis is discussed.
\end{abstract}

\section{INTRODUCTION}

Ansamitocins, new antitumour antibiotics belonging to the family of ansamycins, have specific actions against microtubule systems in eukaryotic cells (Higashide et al., 1977). We have reported that ansamitocins are potent inhibitors of the ciliated protozoon Tetrahymena pyriformis $\mathrm{W}$ and prevent the regeneration of its cilia, which are motile organelles having microtubules as their major functional structures (Tanida et al., 1979). These findings strongly suggest that ansamitocins specifically interact with the ciliary microtubules in $T$. pyriformis.

The present paper deals with the induction of morphological alteration and synchronous cell division in $T$. pyriformis by ansamitocins.

\section{METHODS}

Cultural conditions. Tetrahymena pyriformis strain $\mathrm{W}$ was obtained from Dr Ueda (Kyoto Prefectural University of Medicine, Kyoto, Japan). The organism was grown at $28^{\circ} \mathrm{C}$ in $20 \mathrm{ml}$ of medium containing proteose-peptone $(2 \%, \mathrm{w} / \mathrm{v})$, yeast extract $(0 \cdot 1 \% \mathrm{w} / \mathrm{v})$ and glucose $(0 \cdot 2 \%, \mathrm{w} / \mathrm{v})$, in $250 \mathrm{ml}$ Erlenmyer flasks. It was maintained in $10 \mathrm{ml}$ of this medium in a test tube $(15 \times 160 \mathrm{~mm})$ at room temperature and subcultured every other week.

Ansamitocin treatment. Toa T. pyriformis culture which had been grown for $30 \mathrm{~h}$, ansamitocin was added at a final concentration of $5 \mu \mathrm{g} \mathrm{ml}^{-1}$ and the culture was incubated for a further 5 to $14 \mathrm{~h}$. The cells in $20 \mathrm{ml}$ of culture were harvested by centrifugation $\left(200 \mathrm{~g}, 3 \mathrm{~min}, 20^{\circ} \mathrm{C}\right)$ and then suspended in $40 \mathrm{ml}$ of medium prewarmed to $28^{\circ} \mathrm{C}$. The suspension was cultured at $28^{\circ} \mathrm{C}$ without shaking. Cell density in $1 \mathrm{ml}$ samples, after fixing with glutaraldehyde, was determined with a Celloscope electronic particle counter model 302 (AB Lars Ljungberg, Sweden). Division indices were estimated visually with a phase contrast microscope $(\times 100$ magnification $)$. 

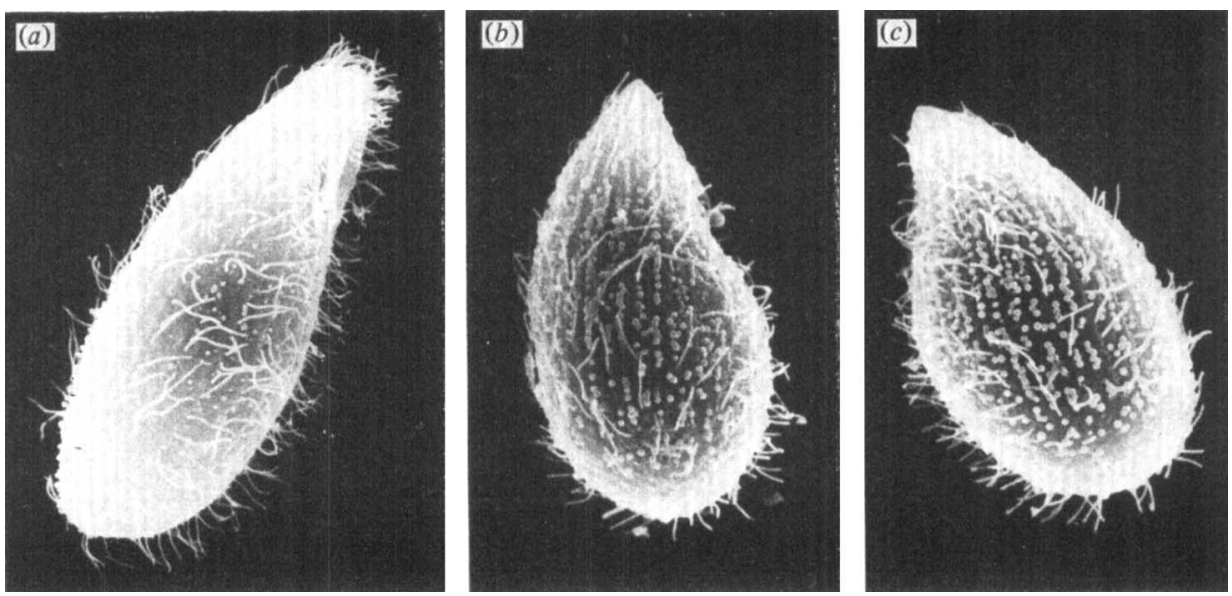

Fig. 1. Alteration of cell morphology of $T$. pyriformis by ansamitocins. Normal cells and cells which had been exposed to ansamitocin P-3 or P-4 $\left(5 \mu \mathrm{g} \mathrm{ml}^{-1}\right)$ for $14 \mathrm{~h}$ were fixed with glutaraldehyde and examined by scanning electron microscopy. (a) Normal cell; (b) cell exposed to ansamitocin P-3; $(c)$ cell exposed to ansamitocin P-4. Magnification $\times 750$.

Use of inhibitors. To avoid non-specific actions, concentrations of inhibitors, except methotrexate, were determined by a broth dilution method (Tanida et al., 1979). Chromomycin $\mathbf{A}_{3}$ and cycloheximide (both at 10 $\mu \mathrm{g} \mathrm{ml}^{-1}$ ) were used as specific inhibitors of RNA and protein syntheses, respectively. Methotrexate (0.05 mM) plus uridine ( $5 \mathrm{~mm}$ ) was used as a specific inhibitor of DNA synthesis in $T$. pyriformis (Zeuthen, 1968). In the experiment concerned with time of addition of inhibitors, $10 \mu \mathrm{l}$ of the inhibitors were added in $1 \mathrm{ml}$ samples. After glutaraldehyde fixation at $200 \mathrm{~min}$, the cell density was determined.

$D N A$ assays. Cells in $20 \mathrm{ml}$ samples were harvested by centrifugation $\left(3000 \mathrm{~g}, 10 \mathrm{~min}, 0{ }^{\circ} \mathrm{C}\right)$ and stored at $-20^{\circ} \mathrm{C}$ until used. The cell pellets were suspended in $1 \mathrm{ml}$ cold $5 \%(\mathrm{w} / \mathrm{v})$ trichloroacetic acid, disrupted by four cycles of freezing and thawing and then the DNA was extracted by the method of Villadsen \& Zeuthen (1970). Concentrations of DNA were determined by the method of Burton $(1956,1968)$ with calf thymus DNA as standard.

Scanning electron microscopy. Tetrahymena pyriformis cells fixed with $0 \cdot 1 \%$ glutaraldehyde were dehydrated through an ethyl alcohol series. For scanning electron microscopy the cells were critical-point dried, coated with gold and viewed with a MSM4C-101 scanning electron microscope.

Chemicals. Ansamitocins P-3 and P-4 were prepared in our laboratories. Chromomycin $\mathbf{A}_{\mathbf{3}}$, cycloheximide, methotrexate and calf thymus DNA were obtained from Takeda Chemical Industries (Osaka, Japan), Sigma, Lederle Laboratories (N.J., U.S.A.) and PL Biochemicals (Milwaukee, Wis., U.S.A.), respectively.

\section{RESULTS}

\section{Alteration of cell morphology during ansamitocin inhibition}

Ansamitocins inhibit the growth of $T$. pyriformis strain $\mathrm{W}$ at low concentrations (Tanida et al., 1979). Increase in cell numbers was stopped at $60 \mathrm{~min}$ after the addition of $5 \mu \mathrm{g}$ ansamitocin P-3 ml-1 to a culture growing exponentially. Arrested cells showed an altered cell morphology (Fig. 1). After $14 \mathrm{~h}$ exposure to ansamitocin P-3 $\left(5 \mu \mathrm{g} \mathrm{ml}^{-1}\right)$, most of the arrested cells became round (Fig. 2). If rounding of cells is estimated as the ratio of breadth/ length, the average value for ansamitocin-arrested cells was 0.62 while that for normal cells was $0 \cdot 45$. Although the ansamitocins caused rounding of the cells, they did not affect their motility at the concentrations used.

\section{Synchronous cell division after removal of ansamitocins}

When cells were exposed to $5 \mu \mathrm{g}$ ansamitocin P-3 ml $\mathrm{m}^{-1}$ for $5 \mathrm{~h}$ or more and then harvested by low-speed centrifugation, synchronous cell division with a high degree of synchrony of 


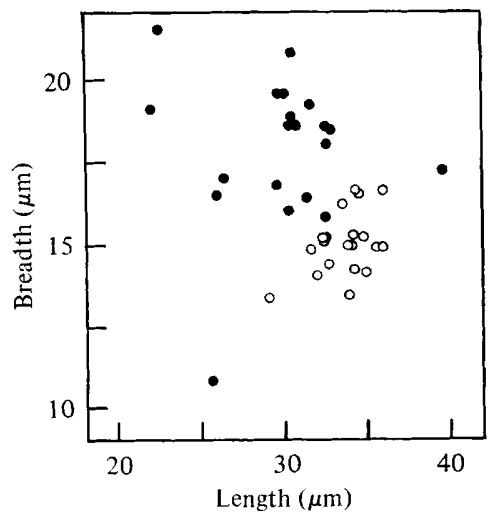

Fig. 2. Size distribution of T. pyriformis cells exposed to ansamitocin P-3 (5 $\left.\mu \mathrm{g} \mathrm{ml}^{-1}\right)$ for $14 \mathrm{~h}$ (O). Breadths and lengths of 20 cells were measured from their photo-image. Results for untreated exponentially growing cells are also shown (O).

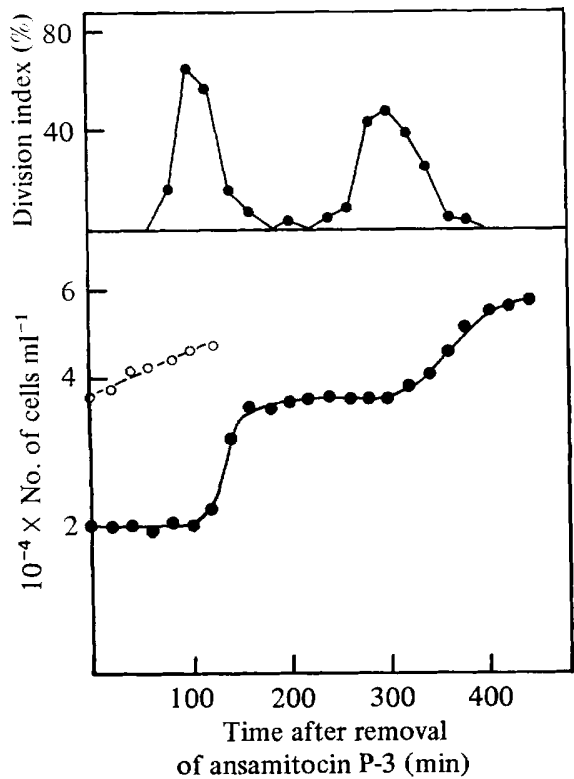

Fig. 3

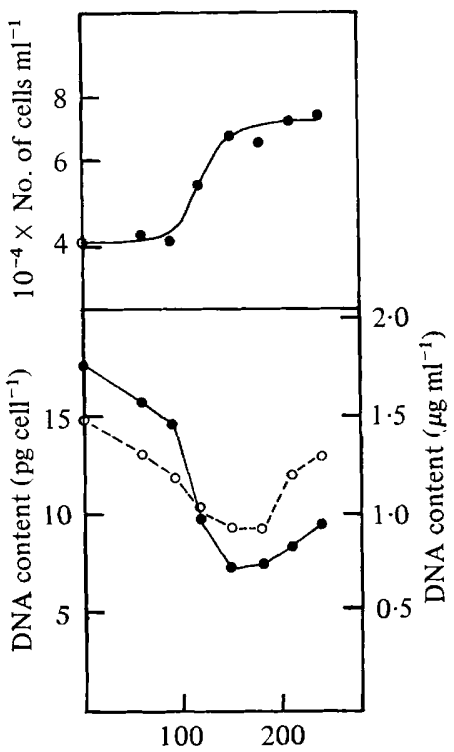

Time after removal of ansamitocin P-3 ( $\mathrm{min})$

Fig. 4

Fig. 3. Synchronous growth of ansamitocin-treated $T$. pyriformis. Cells were exposed to ansamitocin P-3 $\left(5 \mu \mathrm{g} \mathrm{ml}^{-1}\right)$ for $14 \mathrm{~h}$. After removal of the ansamitocin by low-speed centrifugation, cells were suspended in prewarmed medium without antibiotic. At intervals, $1 \mathrm{ml}$ samples were taken and fixed with glutaraldehyde, and the cells were counted as described in Methods (O). Results for cultures without ansamitocin treatment are also shown $(\bigcirc)$. Each point is the mean of five experiments.

Fig. 4. DNA content in ansamitocin-induced synchrony of $T$. pyriformis. The synchronous culture was obtained as described in the legend to Fig. 3. DNA was extracted from cells in $20 \mathrm{ml}$ samples after glutaraldehyde fixation: results are expressed as pg DNA cell ${ }^{-1}(O)$ and $\mu \mathrm{g} \mathrm{DNA} \mathrm{ml^{-1 }}(\bigcirc)$. Each point is the mean of three experiments. 


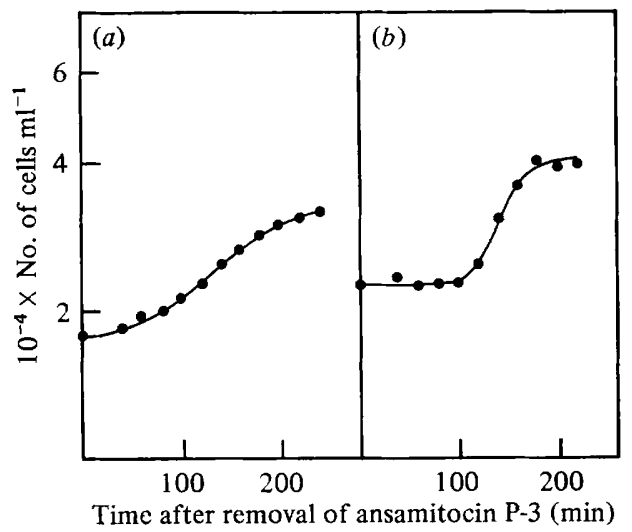

Fig. 5. Effect of time of exposure to ansamitocin on synchronous growth of $T$. pyriformis. An exponentially growing culture was exposed to ansamitocin P-3 $\left(5 \mu \mathrm{g} \mathrm{ml}^{-1}\right)$ for $3 \mathrm{~h}(a)$ or $5 \mathrm{~h}(b)$; cells were then harvested and suspended in prewarmed medium, and cell numbers were determined as described in Methods. Each point is the mean of three experiments.

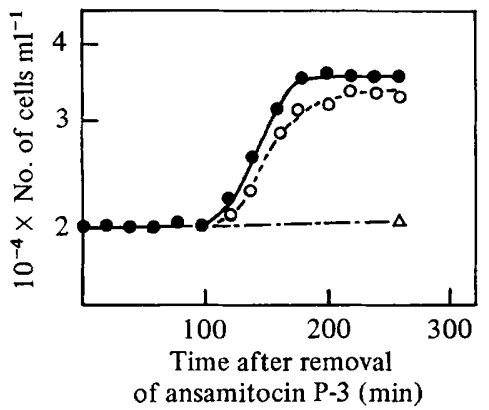

Fig. 6

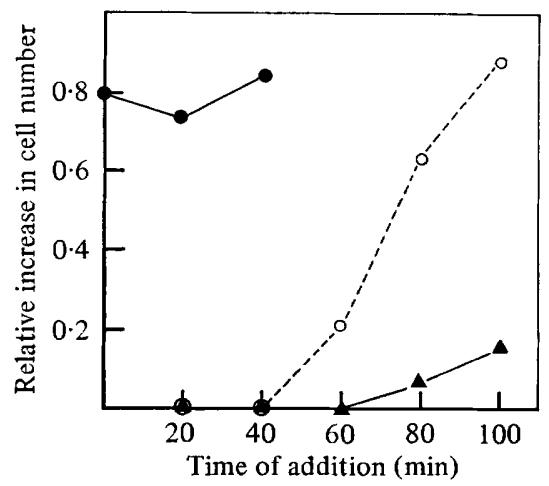

Fig. 7

Fig. 6. Effect of inhibitors on ansamitocin-induced synchrony in $T$. pyriformis. The synchronous culture was obtained as described in the legend to Fig. 3. The following compounds were added at zero time: $O$, methotrexate $(0.05 \mathrm{~mm})$ plus uridine $(5 \mathrm{~mm}) ; \triangle$, chromomycin $\mathrm{A}_{3}\left(10 \mu \mathrm{g} \mathrm{ml}^{-1}\right)$ or cycloheximide $\left(10 \mu \mathrm{g} \mathrm{ml}^{-1}\right)$; no addition. Each point is the mean of four experiments.

Fig. 7. Effect of time of addition of inhibitors on ansamitocin-induced synchrony in T. pyriformis. Each compound was added at $20 \mathrm{~min}$ intervals in $1 \mathrm{ml}$ samples to ansamitocin-treated cultures. Cells were fixed with glutaraldehyde at 200 min after removal of ansamitocin P-3 and counted as described in Methods. The increase in cell number was estimated relative to that in drug-free controls. Inhibitors: 0 , methotrexate $(0.05 \mathrm{~mm})$ plus uridine $(5 \mathrm{~mm}) ; \bigcirc$, chromomycin $\mathrm{A}_{3}\left(10 \mu \mathrm{g} \mathrm{ml}^{-1}\right)$; $\Delta$, cycloheximide $\left(10 \mu \mathrm{g} \mathrm{ml}^{-1}\right)$. Each point is the mean of four experiments.

cytokinesis occurred in the fresh medium without the antibiotic. After $14 \mathrm{~h}$ exposure, there was a first burst of division with a maximum division index of $70 \%$ at 100 min after removal of the antibiotic (Fig. 3). The alteration of the cell shape was restored by the completion of the first division. A second burst of division occurred after 300 min with a peak division index of $55 \%$. The time interval between the two bursts of division was approximately equal to the natural generation time of about $180 \mathrm{~min}$. The DNA content of the cells did not increase till after the completion of the first division (Fig. 4). When cells were exposed to ansamitocin P-3 for $3 \mathrm{~h}$, little synchrony was obtained (Fig. $5 \mathrm{a}$ ); on the other hand, $5 \mathrm{~h}$ exposure caused a burst of synchronous division (Fig. $5 b$ ). 


\section{Effect of inhibitors on the first burst of synchronous division}

In order to clarify the involvement of the syntheses of cellular macromolecules in the first burst of synchronous division, the effect of specific inhibitors was examined. When added at the time of removal of ansamitocin P-3 (time zero), chromomycin $\mathrm{A}_{3}$ and cycloheximide completely suppressed the first division whereas methotrexate plus uridine had only a slight effect (Fig. 6). When inhibitors were added at different times after the removal of ansamitocin P-3, followed by fixing with glutaraldehyde at $200 \mathrm{~min}$, different inhibitory patterns were observed. Complete inhibition by chromomycin $\mathrm{A}_{3}$ was obtained when it was added at 40 min after removal of ansamitocin P-3 and the relative increase in cell numbers reached only about $20 \%$ of the control value when it was added at $60 \mathrm{~min}$ (Fig. 7). On the other hand, suppression by cycloheximide continued throughout every stage before the first division. These results indicate that RNA synthesis in ansamitocin-induced synchrony reaches completion at about $80 \mathrm{~min}$ after removal of the antibiotic while protein synthesis occurs at a late stage or during every stage before the first division. Furthermore, the division may not require the initiation of chromosome replication. In all the experiments described above, no significant difference was observed between ansamitocins P-3 and P-4.

\section{DISCUSSION}

The ciliate protozoon $T$. pyriformis has been used for studies on microtubule systems. In our present studies, ansamitocins caused a rounding of the cell body of $T$. pyriformis $\mathrm{W}$ and inhibited cell division. The former effect appeared to be dependent on the specific action of the ansamitocins because chromomycin $\mathrm{A}_{3}$, cycloheximide and methotrexate plus uridine caused no morphological alteration. The change in shape may have been due to disruption of cortical microtubules, which could be responsible for form maintenance in $T$. pyriformis and be rather more susceptible than ciliary microtubules to ansamitocins. The rounded cells were still motile and had numerous cilia on their surface. In general, microtubules of cilia and flagella are relatively insensitive to antimitotic agents but their formation or regeneration are highly susceptible (Rannestad, 1974; Rosenbaum \& Carlson, 1968; Rosenbaum et al., 1969; Stephens \& Edds, 1976; Tanida et al., 1979). Kennedy \& Zimmerman (1970) have reported that high hydrostatic pressure readily disrupts the cortical microtubules, resulting in a rounding of the cell body, but that such pressures have minimal effect on ciliary structure. Our findings are consistent with these observations.

Tetrahymena pyriformis cells inhibited by ansamitocins were capable of growing with synchronous cell division without active DNA synthesis, but synthesis of both RNA and protein was necessary for ansamitocin-induced synchronous division (Fig. 6). These results indicate that ansamitocins arrest the growth of $T$. pyriformis after the DNA synthesis phase of its cell cycle, and also suggest that the antibiotics do not significantly inhibit DNA synthesis in the ciliate. The cells appeared to continue to synthesize DNA to a certain extent during the period of ansamitocin inhibition, resulting in its accumulation (Fig. 4); this result is similar to that observed for vinblastine inhibition (Stone, 1968a,b). The mitotic spindle one of the most important microtubule systems in eukaryotic cells, plays an essential role in the partition of chromosomes during mitotic division, and the formation of the mitotic spindle is highly susceptible to antimitotic agents. On the basis of their antitubulinic properties (Higashide et al., 1977; Tanida et al., 1979), it is suggested that ansamitocins may interfere with the function of the mitotic spindles in T. pyriformis nuclei, resulting in reversible inhibition of the cell division at metaphase.

Many methods have been developed for the preparation of synchronous cultures of $T$. pyriformis (Anderson et al., 1975; Everhart, 1972). The most extensively used have been heat-shock procedures. Several drugs have been shown to act as reversible inhibitors of division in T. pyriformis and to induce division synchrony. Wunderlich \& Peyk (1969) have demonstrated that colchicine or colcemid inhibit macronuclear division in T. pyriformis; 
the cells recover from the inhibition after a few hours and divide synchronously. Stone $(1968 b)$ has reported that vinblastine-treated $T$. pyriformis is capable of growing synchronously and the division index depends on the duration of the exposure time, a longer exposure resulting in a higher division index. Sedgley \& Stone (1969) have demonstrated that DNA synthesis continues during vinblastine inhibition and additional DNA synthesis following release from vinblastine is not required for the first synchronous division. In our studies, good synchrony was obtained in ansamitocin-treated cultures of $T$. pyriformis. Using the synchrony index $(F)$ of Blumenthal \& Zahler $(1962)$, the degree of synchrony of the first division after removing the antibiotics was $0 \cdot 65$, assuming a normal generation time of $180 \mathrm{~min}$. Ansamitocin-induced division synchrony is probably due to the same mechanism as that of colchicine or vinblastine because of its specific properties with respect to tubulin. In ansamitocin-induced synchrony, cells can synthesize DNA under the influence of ansamitocins. This finding is similar to that for vinblastine. There is a possibility that ansamitocins act as inhibitors of DNA synthesis since maytansine (Kupchan et al., 1972), which is structurally and functionally very similar to ansamitocins (Higashide et al., 1977), inhibits DNA synthesis in murine leukaemia cells (Wolpert-DeFillipes et al., 1975) and reacts with DNA in vitro (Lown et al., 1977). However, our results show that ansamitocins arrest the division of $T$. pyriformis after the DNA synthesis phase in its cell cycle. It is therefore presumed that ansamitocin inhibition of cell division in T. pyriformis is mediated through an inhibition of the function of cellular microtubules, especially mitotic spindles in macroand/or micronuclei in the ciliate. This presumption is supported by observations in mammalian cells (Higashide et al., 1977).

\section{REFERENCES}

Anderson, H. A., Rasmussen, L. \& Zeuthen, E. (1975). Cell division and DNA replication in synchronous Tetrahymena cultures. Current Topics in Microbiology and Immunology 72, 1-20.

Blumenthal, L. K. \& Zahler, S. A. (1962). Index for measurement of synchronization of cell population. Science 135, 724.

Burton, K. (1956). A study of the conditions and mechanism of the diphenylamine reaction for the colorimetric estimation of deoxyribonucleic acid. Biochemical Journal 62, 315-323.

BurToN, K. (1968). Determination of DNA concentration with diphenylamine. Methods in Enzymology 12B, 163-166.

EVERHART, L. P., JR (1972). Methods with Tetrahymena. Methods in Cell Physiology 5, 219-288.

Higashide, E., Asai, M., Ootsu, K., Tanida, S., Kozai, Y., Hasegawa, T., Kishi, T., Sugino, Y. \& YonedA, M. (1977). Ansamitocins, a novel group of maytansinoid antibiotics with antitumor properties. Nature, London 270, 721-722.

KenNedy, J. R. \& Zimmerman, A. M. (1970). The effects of high hydrostatic pressure on the microtubules of Tetrahymena pyriformis. Journal of Cell Biology 47, 568-576.

Kupchan, S. M., Komoda, Y., Court, W. A., Thomas, J. G., Smith, R. M., Karin, A., Gilmore, C. J., Haltiwanger, R. C. \& Bryan, R. F. (1972). Maytansine, a novel antileukemic ansa macrolide from Maytenus ovatus. Journal of the American Chemical Society 94, 1354-1356.

Lown, J. W., Majumdar, K. C., Meyers, A. I. \& Hecht, A. (1977). Studies related to antitumor antibiotics. X. Reactions of maytansine and its analogs with DNA in vitro. Bioorganic Chemistry 6, 453-463.
RANnestad, J. (1974). The regeneration of cilia in partially deciliated Tetrahymena. Journal of Cell Biology 63, 1009-1017.

Rosenbaum, J. L. \& Carlson, K. (1968). Cilia regeneration in Tetrahymena and its inhibition by colchicine. Journal of Cell Biology 40, 415-425.

Rosenbaum, J. L., Moulder, J. F. \& Ringo, B. L. (1969). Flagella elongation and shortening in Chlamydomonas. The use of cycloheximide and colchicine to study the synthesis and assembly of flagella proteins. Journal of Cell Biology 41, 600619.

Sedgley, N. N. \& Stone, G. E. (1969). DNA synthesis in vinblastine synchronized Tetrahymena. Experimental Cell Research 56, 174-177.

Stephens, R. E. \& Edds, K. R. (1976). Microtubules: structure, chemistry and function. Physiological Reviews 56, 709-777.

STONE, G. E. (1968a). Method for reversible inhibition of cell division in Tetrahymena pyriformis using vinblastine sulfate. Methods in Cell Physiology 3, 161-170.

Stone, G. E. (1968b). Synchronized cell division in Tetrahymena pyriformis following inhibition with vinblastine. Journal of Cell Biology 39, 556-563.

Tanida, S., Higashide, E. \& Yoneda, Y. (1979). Inhibition of cilia regeneration of Tetrahymena by ansamitocins, new antitumor antibiotics. Antimicrobial Agents and Chemotherapy 16, 101-103.

VILlADSEN, I. S. \& ZEUTHEN, E. (1970). Synchronization of DNA synthesis in Tetrahymena populations by temporary limitation of access to thymine compounds. Experimental Cell Research 61, 302310.

Wolpert-DeFillipes, M. K., Adamson, R. H., CYSYK, R. L. \& JoHns, D. G. (1975). Initial 
studies on the cytotoxic action of maytansine, a novel ansa macrolide. Biochemical Pharmacology 24, 751-754.

Wunderlich, F. \& PeYK, D. (1969). Antimitotic agents and macronuclear division of ciliates. Experimental Cell Research 57, 142-144.
Zeuthen, E. (1968). Thymine starvation by inhibition of uptake and synthesis of thymine compounds in Tetrahymena. Experimental Cell Research 50, 37-46. 